\title{
REVISTAS MONOGRÁFICAS SOBRE NIETZSCHE
}

Assim Falou NietzSCHE I, 1999. BARRENECHEA, M. A. de y NETO, J.O.P. (org). Letras/UFOP, Rio de Janeiro, 1999. 200 pp. www.7letras.com.br

José Luiz Furtado: A essência da vida na filosofia da arte em $O$ nascimento da tragédia./ Maria Cristina Franco Ferraz: Nietzsche: Filosofia e paródia./ Mauro Lúcio Leitão Condé: Nietzsche e Wittgenstein: semelhanças de família./ Roberto Charles Feitosa de Oliveira: Ex-verter a metafísica: a questão do sensivel em Nietzsche e Heidegger./ Roberto Machado: Zarastutra, o apolíneo e o dionisíaco./ Rodrigo Duarte: Adorno e Nietzsche: aproximações./ Rosa Maria Dias: Nietzsche e a questão do gênio./ Alexandre Mendonça: Ciência e paródia no eterno retorno de Nietzsche./ Daniel Lins: A escrita das origens: Artaud e Nietzsche./ Imaculada Kangussu: Palavras sonantes, verdades e mentiras./ Miguel Angel de Barrenechea: Ecce Homo: a arte de "chegar a ser o que se é"./ Nina Reis Saroldi: O que fazer para viver bem com o mal? ( $O$ sofrimento em Friedrich Nietzsche)./ Olímpio José Pimenta Neto: Nietzsche naturalista? / Ricardo José Camêlo da Silva: Nietzsche e a questão do conhecimento./ Rosana Suarez: Platão professor aos olhos do professor Nietzsche./ Viviane Mosé: Nietzsche e a genealogia do sujeito.

Assim Falou Nietzsche II, 2000. Memória, Tragédia e Cultura, Relume-Dumará, Rio de Janeiro, pp. 169. BARRENECHEA, M. A. de y NETO, J.O.P. (Org). Letras/UFOP, Rio de Janeiro, 1999. 200 pp. www.7letras.com.br

Charles Feitosa: Da utilidade do esquecimento para a filosofia./ Daniel Lins: Nietzsche e Artaud: escrita e poética do túmulo./ Gilvan Fogel: Fragmento de interpretação de «do conhecimento imaculado», in: Zaratustra II./ Maria Cristina Franco Ferraz: Teatro e máscara no pensamento de Nietzsche./ Marta Luzie de Oliveira Frecheiras Civale: A questão do ressentimento em Nietzsche e Max Scheller./ Miguel Angel de Barrenechea: Tragédia hoje: a contemporaneidade do arcaico./ Miguel Antonio do Nascimento: Contribuição à leitura de Nietzsche./ Olímpio José Pimenta Neto: Arte e conhecimento em Nietzsche./ Oswaldo Giacoia Junior: Genealogia da moral e arqueologia da cultura./ Paulo Pinheiro: Prolegômenos a uma história nietzschiana da arte./ Rosa Maria Dias: Nietzsche e Wagner: "amizade de astros»./ Scarlett Marton: Só acreditaria num deus que soubesse dançar./ Valéria Cristina Lopes Wilke: Memória-esquecimento: Nietzsche e Benjamin.

Assim FaLou Nietzsche III, 2001. Para uma filosofia do futuro, 7 Letras, Rio de Janeiro, 2001. 348 pp. . BARRENECHEA, M. A. de , CASANOVA, M.A., DIAS, R. M. y FEITOSA y NETO, J.O.P. (Org). www.7letras.com.br André Martins: Nietzsche, Espinosa, o acaso e os afetos - encontros entre o trágico e o conhecimento intuitivo./ Bernardo Barros Coelho de Oliveira: $O$ último narrador: 
considerações a partir de Nietzsche, Benjamin, Adorno e Handke./ Charles Feitosa: Por que a filosofia esqueceu a dança? / Claudia Drucker: Nietzsche e a necessidade de redenção./ Daniel Lins: Nietzsche e Artaud: por uma exigência ética da crueldade./ Gilvan Fogel: Vida como um jogo de mando e de obediência./ Jason Wirth: Desejo animal: Nietzsche, Bataille e um mundo sem imagem./ José Thomaz Brum: Nietzsche e Schopenhauer - da admiração à decepção./ Katia Muricy: A arte do estilo./ Marcia Sá Cavalcante Schuback: Sem título./ Marco Antonio Casanova: Experiência e niilismo: Nietzsche e o problema do devir./ Maria Cristina Franco Ferraz: Por uma filosofia para orelhas pequenas./ Miguel Angel de Barrenechea: Nietzsche: para uma nova era trágica./ Miguel Antonio do Nascimento: O tempo e a moral - problema para a "filosofia do futuro"./ Mónica B. Cragnolini: Do corpo-escrita.Nietzsche, seu "eu” e seus escritos./ Olímpio José Pimenta Neto: Filosofia para o Brasil./ Oswaldo Giacoia Junior: Nietzsche: um século depois./ Paulo Cesar Souza: “Gaia Ciência”./ Rosa Maria Dias: Dioniso contra Parsifal./ Scarlett Marton: Assim Silenciou Nietzsche./ Adriany Ferreira de Mendonça: Sócrates e o ressentimento: o crepúsculo de um ídolo./ Adriany e Alexandre Ferreira de Mendonça: Reversão do platonismo: algumas considerações acerca da interpretação deleuziana de Nietzsche./ Ana Cláudia Gama Barreto: O centauro nietzschiano./ Bárbara Lucchesi Ramacciotti: A concepção nietzschiana de corpo enquanto Grande Razão./ Cristina dos Santos Souza: Empedocles: o conhecedor trágico da civilização em estado puro, segundo Friedrich Nietzsche./ Ederson Halair Hammes: A vontade de nada ou notas sobre a concepção de niilismo em Nietzsche./ Fernanda Machado de Bulhões: O enigma de Sócrates: “o abismo mais profundo e a mais alta elevação”./ Gracia Mônica Masselli Hardman Vianna: Como Nietzsche se torna o que se é - considerações em torno de Ecce Homo./ Henry Burnett: Dionísio contra o pessimismo romântico./ Janaína Cardoso de Mello: Matrix: a filosofia futurista de um Zaratustra cibernético./ Jorge Moraes: Nietzsche: a música como filosofia./ Luis Eduardo Rubira: $O$ critério de avaliação nietzschiano./ Luiz Celso Pinho: A estética de Zaratustra./ Marcelo Lion Vilella Souto: As influências de Heráclito na obra de Friedrich Nietzsche./ Márcio Danelon: Nietzsche e a ética do "cuidado de si": uma interpretação à luz de Foucault./ Marcos Sinésio Pereira Fernandes: Alguns aspectos do pensamento da vontade em torno de O nascimento da tragédia de Friedrich Nietzsche./ Marcos Vinicio Guimarães Giusti: A figuração da morte em Nietzsche./ Martha Solange Perrusi: Ilusões da bela aparência diante do horror da existência./ Nina Reis Saroldi: Moral nobre, moral de lobos?/ Rafael Haddock Lobo: A virtude e o outro homem./ Richard Fonseca: Da determinação da vontade enquanto vontade de poder./ Sávio Mangaveira: "O nascimento da tragédia”: um livro anticristão?/ Silvia Pimenta Velloso Rocha: O perspectivismo como doutrina da imanência./ Vânia Dutra de Azeredo: Potência e superação: perspectivas da vontade.

AsSIM FALOU NIETZSCHE IV, 2003. A fidelidade à terra: arte, natureza e política. DP\&A, Rio de Janeiro, 2003. 412 pp. BARRENECHEA, M. A. de, FEITOSA, C. y PINHEIRO, P. (org) www.7letras.com.br 
Oswaldo Giacoia Junior: O indivíduo soberano e o indivíduo moral./ Miguel Angel de Barrenechea: Nietzsche: memória trágica e futuro revolucionário./ Germán Meléndez: $O$ aprofundamento do pessimismo como chave de continuidade no pensamento de Nietzsche./ José Jara: Sobre ensaios e transvalorações./ Rosana Suarez: Aspectos da "mínima política" de Nietzsche./ Cíntia Vieira da Silva: Moral e cultura: ressonâncias e dissonâncias entre Nietzsche e Freud./ Marinete Araujo da Silva: Considerações sobre o pensamento político de Nietzsche./ Rodrigo Guéron: Como Nietzsche compreende "história" e a descrição do "século da história"./ Selmo Gliksman: Os fins do homem e o advento do sobre-humano./ Vicente Eudes Veras da Silva: Do Übermensch ao homem tecnologizado: uma redefinição de nossa humanidade./Willis Santiago Guerra Filho: A última aventura humana sobre a terra./ Maria Cristina Franco Ferraz: Da valorização estratégica da metáfora em Nietzsche./ Olímpio José Pimenta Neto: Ainda sobre Nietzsche e a verdade./ Carole Gubernikoff: Zarastutra cantor: uma leitura a partir de "O convalescente" de Assim Falou Zaratustra./ Paulo Pinheiro: Drama e fidelidade em Nietzsche./ Rosa Maria Dias: Nietzsche e Schopenhauer: uma primeira ruptura./ Fernanda Machado de Bulhões: Arte, razão e mistério na filosofia "pré-socrática”./ Marcos Sinésio Pereira Fernandes: $O$ dionisismo segundo o pensamento de Nietzsche em torno de O nascimento da tragédia./ Paula Braga: Os parangolés de Hélio Oiticica: Nietzsche entra no samba./ Rafael Haddock Lobo: Sentido da terra, vida e arte: perspectivas de um radicalismo estético em Nietzsche./ Rogério Lopes: Entre ensaio e aforismo: notas sobre o modo de apresentação dos argumentos na filosofia de Nietzsche./ Mónica B. Cragnolini: Os caminhos do sentido: entre um Nietzsche heideggeriano e um Nietzsche derridiano./ Daniel Lins: A história da cultura é a história da crueldade./ Charles Feitosa: O mal do mar em Nietzsche./ Marco Antonio Casanova: A religião da terra: o lugar do sagrado no pensamento de Friedrich Nietzsche./ David Lapoujade: Sobre a passividade./ Marcelo Lion Villela Souto: Apontamentos sobre as ressonâncias entre Nietzsche e Heráclito./ Maria Cristina dos Santos de Souza: Tales: a descoberta do princípio da unidade grega, segundo Friedrich Nietzsche./ Maria Eugênia de la Roca: A crítica nietzschiana do conceito de identidade./ Richard Fonseca: Ciência enquanto interpretação no pensamento de Nietzsche./ Roberto Saraiva Kahlmeyer-Mertens: Nietzsche: metafísica, errância e subjetividade./ Virginia Susana de Azeredo França: Positividade e negatividade da crueldade em Nietzsche.

CADERNos NIETZSCHE. Revista del GEN (Grupo de Estudos Nietzsche). No. 16, 2004, Sao Paulo. Brasil. Directora Scarlett Marton, Departamento de Filosofia - USP / Av. Prof. Luciano Gualberto, 315 - sala 1005 / 05508-900 - Sao Paulo - SP - Brasil. gen@edu.usp.br - www.fflch.usp.br/df/gen.htm

Eric Blondel, Nietzsche: a vida e a metáfora / Anna Hartmann Cavalcanti, Eduard Hanslick / Jarlee Oliveira Silva Salviano, Schopenhauer, Nietzsche e a crítica da filosofia universitária /Nuno Venturinha, Sobre a Metamorfoseabilidade da Experiência em Die Geburt der Tragödie de Nietzsche / Marco Parmeggiani, Nietzsche: o pluralismo e a pós-modernidade. 
Estudios Nietzsche. Nietzsche Y el LenGUAJE. Revista de la Sociedad Española de Estudios sobre F. Nietzsche (SEDEN). N 4, 2004. Director: Luis E. De Santiago Guervós, Departamento de Filosofía, Universidad de Málaga / Campus de Teatinos/ 29071 Málaga - España. lesantiago@uma.es - www.estudiosnietzsche.org Babette Babich, Música y palabras en Nietzsche:Sobre la cuestión de la ciencia, el estilo y la música de la tragedia griega antigua. / Jesús Conill, La poetización nietzscheana del lenguaje y del pensamiento. / Cirilo Flórez, Retórica, metáfora y concepto en Nietzsche. / Enrique Lynch, La discriminación. Lenguaje, percepción y música a partir de Nietzsche. / Gerd Schank, Herman Siemens, Paul van Tongeren, Procesos semánticos en los textos filosóficos de Nietzsche y su función filosófica: Algunos resultados de la investigación sobre el «Diccionario-Nietzsche». / Vicenzo Vitiello, «En lucha con el lenguaje». De Wittgenstein a Nietzsche./Luis de Santiago Guervós, Informe bibliográfico

ESTUDIOS LIBRES: Paulina Rivero Weber, Nietzsche y la filosofía en México. / Manuel Torres Vizcaya, La ausencia presente de Nietzsche en Ser y tiempo. Proximidad entre el concepto nietzscheano 'interpretación' y el heideggeriano 'comprensión'.

INFORMACIÓN BIBLIOGRÁFICA. Novedades Bibliográficas. Revistas monográficas sobre Nietzsche

Concordancias La voluntad de poder - Edición Colli-Montinari

NOTICIAS: Apuntes póstumos: primavera 1868 - otoño 1869; Comentario crítico al epistolario: enero 1885 - diciembre 1886; Léxico de contemporáneos de Nietzsche

Coloquio de Treilles: Filosofía de la Aurora; Encuentro sobre Heidegger y Nietzsche; IV Jornadas Internacionales Nietzsche 2004

RECENSIONES CRÍTICAS

F. Nietzsche, L'Anticrist. Maledicció sobre el cristianisme, (J.B. Llinres)

L.E. De Santiago Guervós, Arte y poder. Aproximación a la estética de Nietzsche (L. Puelles)

P. Rivero Weber, Nietzsche, verdad e ilusión. Sobre el concepto de verdad en el joven Nietzsche (G. Rivara Kamaji)

E. Ruiz Callejón, Nietzsche y la filosofía práctica. La moral aristocrática como búsqueda de la salud (L.E. De Santiago Guervós)

E. Severino, L'anello del ritorno (F. Morató).

Journal of NiETzSCHE StUdies. 27 (2004). Revista de la Friedrich Nietzsche Society. Editor Prof. Daniel W. Conway, Department of Philosophy, Pennsylvania State University, 240 Sparks Building, University Park, Pa 16802, USA. Nietzsche@psu.edu Beyond a Joke: Nietzsche and the Birth of "Super-Laughter. Subjects: Nietzsche, Friedrich Wilhelm, 1844-1900 - Views on laughter.

Downard, Jeffrey Brian, Nietzsche and Kant on the Pure Impulse to Truth. / Westfall, Joseph, Zarathustra's Germanity: Luther, Goethe, Nietzsche./ Helm, Barbara, Combating Misogyny? Responses to Nietzsche by Turn-of-the-Century German Feminists. / Book Reviews: Picart, Caroline Joan, Nietzsche and "An Architecture 
of Our Minds" (review). / Kostka, Alexandre, ed. Nietzsche and "an architecture of our minds". / Wohlfarth, Irving, ed. Nietzsche, Friedrich Wilhelm, 1844-1900 - Contributions in art and architecture. Murray, Peter Durno, Time-Fetishes: The Secret History of Eternal Recurrence (review). / Santi, Raffaella, Nietzsche, il ribelle aristocratico: Biografia intellettuale e bilancio critico (review). / Losurdo, Domenico. Nietzsche, il ribelle aristocratico: biografia intellettuale e bilancio critico.

Journal of Nietzsche Studies. 28, Autumn, (2004).

Brobjer, Thomas H, Nietzsche's Reading About Eastern Philosophy. / Smith, David (David James), Nietzsche's Hinduism, Nietzsche's India: Another Look. / Moeller, Hans-Georg, The "Exotic" Nietzsche--East and West. / Geir Sigurdsson, Wandering Beyond the Bounds: Nomadism, Health, and Self-Undermining./ Davis, Bret W, Zen After Zarathustra: The Problem of the Will in the Confrontation Between Nietzsche and Buddhism, / Groff, Peter S., Al-Kindi and Nietzsche on the Stoic Art of Banishing Sorrow.

New Nietzsche Studies. The journal of the Nietzsche Society. 6 (2003), Nos 1 \& 2 . Editores David B Allison y Babette E. Babich. Departament of Philosophy, Fordham University, 113 West $60^{\text {th }}$ Street, New York, New York, 10023, USA. babich@fordham.edu

Ecology, Dynamics, Chaos, Nature.: Reinhart Mauer, Ecological Nietzsche? The Will to Power and the Love of Thing. / Ralph Acampora, The Joyful Wisdom of Ecology. / Peter Douglas, Nietzsche and Chaos. / Babette E. Babich, A Philological Note on Nietzsche's Chaos sive natura: Theogony, Genesis, and Playing Stars. / Niels Helsloot, Gaya Scienza: Nietzsche as a Friend. / Paul van Tongeren, "On the Friend" in Nietzsche's Zarathustra. / Alphonso Lingis, The Return to, The Return of Peoples of Long Ago and Far Away.

Center-Feature: earl R. Nitscheke Stamps. Book Rewiew.

Symposium Gary Shapiro, ARCheologies of Vision: Robert Mugerauer, To Love the Earth: The Abysmal Sublime From Landscape to Laughter. / Mark A. Cheetham, LandPhil. / Joel Black, Towards an Iconoclastic Aesthetics: Nonsites and Non-Sense. / Penny Florence, Alcyone on the Jetty or The Lady Vanishes. / Stephen Barker, Turns of Phrase: A Geometry of Shapirean Aesthetics. / Babette E. Babich, Claude Lorraine and Raphael: Shapiro's Archaeology of Transfiguration. / Gary Shapiro, Geometry, Gardens, Gender: Writing Aesthetics after Nietzsche.

NIETZSCHEFORSCHUNG. JAHRBUCH DER NIETZSCHE-GESELLSCHAFT. Bd. 11. Herausgegeben von Volker Gerhardt und Renate Reschke.Akademie Verlag, Berlin 2004, 312 pp. Dirección: Akademie Verlag / Palisadenstr. 40 / D-10243 Berlín. info@akademieverlag.de - akademie-verlag.de ANTIKE UND ROMANTIK BEI NIETZSCHE

Steffen Dietzsch, Karl Joël: Nietzsche und die Romantik neu gelesen. / Dirk von Petersdorff, Nietzsche und die romantische Ironie. / Violetta L. Weibel, Hölderlin und Nietzsche über Philistertum und wahre Bildung. / Justus L. Ulbricht, Neuro- 
mantik - Ein Rettungsversuch der Moderne mit Nietzsche. / Matthew H. Meyer, Die Einheit der Gegensätze als tragisches Prinzip. / Enrico Müller, Politik, Sophistik und Philosophie im Zeitalter der athenischen Demokratie nach Nietzsche. / Ole Schütza, Nietzsche und Thukydides. / Konstantin Broese, Nietzsche und die antike Aufklärung. / Christian Wollek, Nietzsche und das Problem Sokrates. / Hans-Martin Gerlach, Nietzsche im linken und rechten Zerrspiegel. / Volker Caysa, Nietzsches Kritik der Macht und die Macht der Kritik nach Nietzsche. / Erwin Hufnagel, Dèformation professionelle. Zur Rezeption Friedrich Nietzsches und der Phänomenologie Friedrich Schelers Pia Daniela Volz, Wahrsinn oder Wahnsinn? Nietzsche als Objekt elletristischer Begierde.

Nietzsche-Studien. Internationales JahrbuCh FÜr die Nietzsche-ForsChung. 33 (2004). Editado por Günter Abel, Josef Simon, Werner Stegmaier; editado por Walter de Gruyter, Berlín. Redacción: Dr. Ulrich Dirks / Institut für Philosophie / TU Berlin. Sekr. TEL 12/1 / Ernst-Reuter-Platz 7 / D-10587 Berlin. o-journals@ deGruyter.com

Christof Windgätter, Rauschen, Nietzsche und die Materialitäten der Schrift. / Daniel Came, Nietzsche's Attempt at a Self-Criticism: Art and Morality in The Birth of Tragedy. / Michael Skowron, Zarathustra-Lehren. Übermensch, Wille zur Macht, ewige Wiederkunft. / Wener Stegmaier, «Philosophischer Idealismus» und die «Musik des Lebens». Zu Nietzsches Umgang mit Paradoxien. Eine kontextuelle Interpretation des Aphorismus Nr. 372 der Fröhlichen Wissenschaft. I Peter Bornedal, The Incredible Profundity of the Truly Superficial. Nietzsche's 'Master' and 'Slave' as Mental Configurations. / Bernard Bischoff, Nietzsche and the New. / Tsarina Doyle, Nietzsche's Appropriation of Kant. / Ekaterina Poljakova, «Ästhetische Vollendung». Zur philosophischen Ästhetik Nietzsches und Bachtins. / Paul Mirabile, The Nomadic Thought. Friedrich Nietzsche and Zhuang Zi. Convergences and Divergences.

BERICHT: Tanja Zeeb, Die Wirkung Nietzsches auf die deutsche Gesellschaft der Jahrhundertwende im Spiegel der Tagespresse.

Edition und Dokumentation: Johann-Schistoph Emmelius, "Ich hab' mein' Sach' auf nichts gestellt» Zu Herkunft, Funktion und Vorgeschichte eines Zitats in Franz Overbecks Brief an Friedrich Nietzsche vom 15. April 1883.

Miszelle: Joan Stanbugh, All Joys Want Eternity.

Diskussion: Peter Georgsson, Nietzsche's Hammer Again

BEITRÄGE ZUR QUELLENFORSCHUNG: Sören Reuter, Reiz-Bild-Unbewusste Anschauung. Nietzsches Auseinandersetzung mit Hermann Helmholtz' Theorie der unbewussten Schlüsse in Über Wahrheit und Lüge im aussermoralischen Sinne Nachweis: Michael Skowron, Nachweis aus Gladisch, August: Herakleitos und Zoroaster

Rezensionen, Sammelbesprechungen.

elaborado por Luis E. De Santiago Guervós Universidad de Málaga 